\title{
Characteristics of Hybrid Surface Plasmon Polaritons at a Chiral Graphene Metal Interface in Cylindrical Waveguides
}

\author{
Mariam Saeed \\ UAF: University of Agriculture Faisalabad \\ abdul ghaffar ( $\sim$ aghaffar16@uaf.edu.pk) \\ University of Agriculture Faisalabad \\ Majeed A. S. Alkanhal \\ King Saud University \\ Yasin Khan \\ King Saud University
}

\section{Research Article}

Keywords: Surface Plasmon Polaritons, Chiral, Graphene, Waveguide

Posted Date: March 10th, 2021

DOI: https://doi.org/10.21203/rs.3.rs-261771/v1

License: (c) (i) This work is licensed under a Creative Commons Attribution 4.0 International License.

Read Full License 


\title{
Characteristics of Hybrid Surface Plasmon Polaritons at a Chiral Graphene Metal Interface in Cylindrical Waveguides
}

\author{
Mariam Saeed, ${ }^{1}$ Abdul Ghaffar, ${ }^{2}$ Majeed A. S. Alkanhal, ${ }^{3}$ and Yasin Khan ${ }^{4}$ \\ ${ }^{1,2}$ Department of Physics, University of Agriculture, Faisalabad, Pakistan \\ ${ }_{3,4}^{3}$ Department of Electrical Engineering, King Saud University, Riyadh, Saudi Arabia \\ ${ }^{1}$ maryamawan87@yahoo.com,, ," aghaffar16@uaf.edu.pk, ${ }^{3}$ majeed@ksu.edu.sa, \\ ${ }^{4}$ yasink@ksu.edu.sa
}

Corresponding Author: aghaffar16@uaf.edu.pk

\begin{abstract}
A detailed theoretical analysis of the hybrid surface plasmon mode generation and propagation at a chiral graphene metal (CGM) interface for a cylindrical structure is presented. The conductivity of graphene is modeled using the Kubo formalism and the dispersion relation for the hybrid surface waves is computed on the basis of the Kubo formalism and impedance matching boundary conditions. The hybrid mode consisting of lower and upper plasmon modes is witnessed due to the presence of the chiral medium. The frequency band gap between the lower and upper plasmon modes is found to be sensitive and tunable with respect to the chiral strength, radial distance of the waveguide, and chemical potential of graphene. The propagation length and effective refractive index can also be modulated by varying the chiral strength and chemical potential. For very high values of chirality and biasing voltage, the Goos-Hänchen effect is observed at the CGM interface. The cutoff values of chiral strength as a function of normalized frequency make the proposed structure applicable for chiroptical and chemical sensing and enantiomeric detection in the $\mathrm{THz}$ frequency regime.
\end{abstract}

Key Word: Surface Plasmon Polaritons, Chiral, Graphene, Waveguide

\section{Introduction}

Chiral-filled plasmonic waveguides have drawn the attention of the research community in recent years due to their ability to act as sensors in various fields [1]. Chirality is ubiquitously present in nature, from the largest objects, such as galaxies, to the tiniest objects, such as atoms. In a biological system, this crucial phenomenon is responsible for the development of organs and shapes with perfect symmetry. The color of insects and the fragrance of fruits are governed by the chirality of chemical elements called enantiomers. These enantiomers play a key role in the production of drugs, as the chiral behavior of molecules makes them beneficial or dangerous for health. The optical response of enantiomers in terms of the optical rotation of light provides an opportunity to determine the chiral behavior, concentration, and purity of an enantiomeric chemical or protein for chemical and biological sensing [2]. Polarization is one of the basic wave properties of light that governs the interaction of light and optically active substances.

The interaction of light with metal or dielectric surfaces excites surface plasmon polaritons (SPPs), leading to the coupling of polarized light with freely oscillating electrons at the metal-air or metal-dielectric interface [3]. The electromagnetic field enhancement by the propagating SPPs and the absorption of light waves in the form of localized SPPs are significantly altered by the properties of the adjoining materials of the interface, enabling plasmonic waveguides for sensing 
applications [1] in addition to various communication [4], optoelectronic [5], and THz applications [6]. The SPP waveguide sensors are usually composed of thin metal film operationalized by a dielectric layer [7]. Any change in the refractive index of the dielectric layer adjoining the metal surface due to the binding reaction of the dielectric with chemicals or biomolecules is identified by the variation in the propagation behavior of the surface waves. Optical sensing can also be achieved by using optically active chiral materials as analytes. The attachment of chiral molecules with metal surfaces gives rise to circular dichroism, which is greatly enhanced by evanescent field resonances [8]. The sensitivity of the sensor can be modified by modulating the surface waves. For this purpose, graphene is the most suitable candidate, as it provides active control over the propagation of surface waves. The dynamical tuning of the fermi level of graphene provides an additional degree of freedom for the active modulation of the SPPs enhancing circular dichroism for chiral sensing.

Numerous studies based on chiral waveguides having different configurations, such as chiral slabs bounded by a perfect conductor medium [9], planar chiral structures [10], chiral graphene metal (CGM) planar structures [11], chiral-filled double-layer graphene planar structures [12], and chiral-lined metal cylinders [13], have revealed the existence of hybrid plasmon modes instead of only TM or TE modes. This happens due to the coupling between the left-circularly polarized (LCP) and right-circularly polarized (RCP) waves in the chiral medium. A chiral metal planar structure exhibited the generation and propagation of surface waves at the metal interface. The propagation length of the surface waves was found to be sensitive to the chiral strength of the analyte [10]. Both backward and forward propagating modes of surface waves were identified in cylindrical waveguides filled with anisotropic uniaxial chiral material [14]. Circular waveguides with chiral dielectric lining were also found to be supporting hybrid surface waves for broad banding applications [13]. Dispersive behavior sensitive to the chiral strength and thickness of the waveguide core was reported for metal-chiral-metal waveguides [15]. The generation of upper and lower hybrid plasmon modes with band gaps sensitive to the chiral strength and fermi level of graphene was investigated for a planar CGM interface in [16]. Multilayered graphene-chiralgraphene planar waveguides expressed the disappearance of surface waves for certain chiral strengths [12]. The fermi level proved to be an additional factor along with chiral strength for the taming or modulation of the surface waves for on-chip enantiomeric detection and sensing applications. The analysis of the propagation characteristics of hybrid surface plasmon modes for enantiomeric detection at the CGM interface inside a cylindrical waveguide is the goal of the present study.

Surface wave generation and propagation along the circular cylindrical CGM interface is examined in the present study. The next section presents the mathematical formulations for the derivation of the dispersion relation of the hybrid SPPs along the CGM interface. However, the influence of the chiral strength, chemical potential of graphene, radius of the cylinder, and refractive index of the chiral material on the dispersive behavior of surface plasmons is presented by generating and analyzing numerical results in detail in the following section. All the formulations contain $\exp (\mathrm{j} \omega \mathrm{t})$, which represents the time dependence factor.

\section{Formulations and Methodology}

A cylindrical structure consisting of a metal shell having permittivity $\left(\varepsilon_{\mathrm{m}}\right)$ and permeability $\left(\mu_{\mathrm{m}}\right)$ is considered. The core of the structure is filled with an isotropic chiral material of permittivity $\left(\varepsilon_{c}\right)$, permeability $\left(\mu_{c}\right)$, and chirality $(\xi)$. An infinitesimally thin graphene sheet is coated on the 
chiral material and inside the metal surface, as shown in Fig. 1. The permeability of both the metal and chiral material is taken as $\mu_{\mathrm{m}}=\mu_{\mathrm{c}}=\mu_{\mathrm{o}}$. The surface waves propagate at the CGM interface along the $\mathrm{z}$-axis due to cylindrical symmetry. The core region of the cylinder filled with chiral material has $\rho<\mathrm{R}$. However, $\rho>\mathrm{R}$ for the metal-based cladding region. The optical rotation of the chiral material facilitates the coupling of the electric and magnetic fields, triggering hybrid surface waves. This coupling corresponds to the following constitutive relations for electric and magnetic fields: $\mathrm{D}=\varepsilon \mathrm{E}-\mathrm{j} \xi \mathrm{B}$ and $\mathrm{H}=-\mathrm{j} \xi \mathrm{E}+\mathrm{B} / \mu[17,18]$, where the optical rotation is governed by the chiral strength denoted by $\xi$.

To compute the electromagnetic fields for the azimuthally symmetric $\left(\frac{\partial}{\partial \theta}=0\right)$ hybrid mode in each region, the analytical solution of Maxwell equations is obtained using cylindrical coordinates along with suitable boundary conditions. For the core region where $\rho<$ R, the electric and magnetic fields are as follows:

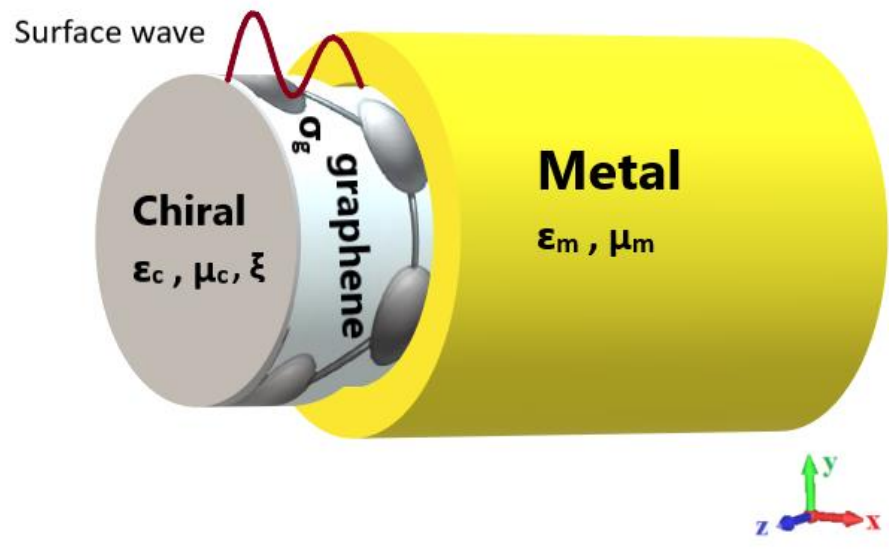

Fig. 1. Surface wave excitation and propagation in CGM-based cylindrical structure.

$$
\begin{aligned}
& E_{z}^{1}=\left[A_{m} I_{m}\left(\gamma_{R} \rho\right)+B_{m} I_{m}\left(\gamma_{L} \rho\right)\right] \exp (j m \theta) \exp (j \beta z) \\
& E_{\varphi}^{1}=-\left[\frac{k_{+}}{\gamma_{R}} A_{m} I_{m}^{\prime}\left(\gamma_{R} \rho\right)-\frac{k_{-}}{\gamma_{L}} B_{m} I_{m}^{\prime}\left(\gamma_{L} \rho\right)\right] \exp (j m \theta) \exp (j \beta z) \\
& E_{\rho}^{1}=-\left[\frac{j \beta}{\gamma_{R}} A_{m} I_{m}^{\prime}\left(\gamma_{R} \rho\right)+\frac{j \beta}{\gamma_{L}} B_{m} I_{m}^{\prime}\left(\gamma_{L} \rho\right)\right] \exp (j m \theta) \exp (j \beta z) \\
& H_{z}^{1}=\frac{j}{\eta_{c}}\left[A_{m} I_{m}\left(\gamma_{R} \rho\right)-B_{m} I_{m}\left(\gamma_{L} \rho\right)\right] \exp (j m \theta) \exp (j \beta z) \\
& H_{\varphi}^{1}=-\frac{j}{\eta_{c} \sqrt{1+\chi^{2}}}\left[\frac{k_{+}}{\gamma_{R}} A_{m} I_{m}^{\prime}\left(\gamma_{R} \rho\right)+\frac{k_{-}}{\gamma_{L}} B_{m} I_{m}^{\prime}\left(\gamma_{L} \rho\right)\right] \exp (j m \theta) \exp (j \beta z) \\
& H_{\rho}^{1}=\frac{1}{\eta_{c} \sqrt{1+\chi^{2}}}\left[\frac{\beta}{\gamma_{R}} A_{m} I_{m}^{\prime}\left(\gamma_{R} \rho\right)-\frac{\beta}{\gamma_{L}} B_{m} I_{m}^{\prime}\left(\gamma_{L} \rho\right)\right] \exp (j m \theta) \exp (j \beta z)
\end{aligned}
$$

where $\gamma_{R, L}=\sqrt{\left(\beta^{2}-k_{ \pm}^{2}\right)}$ is the azimuthal propagation constant, $\eta_{c}=\sqrt{\frac{\mu_{c}}{\varepsilon_{c}}}$ is the impedance of the chiral material, and $\chi=\xi / \eta_{c}$ is the normalized chirality admittance. The wave numbers of the 
$\mathrm{RCP}$ and LCP waves are represented by $k_{+}$and $k_{-}$, respectively, with their corresponding mathematical expressions as $k_{ \pm}=n_{c}\left( \pm \chi+\sqrt{\left(1+\chi^{2}\right)}\right) k_{o}$, where $k_{o}=\omega \sqrt{\mu_{o} \varepsilon_{o}} . \quad I_{m}\left(\gamma_{R, L} \rho\right)$ signifies the modified Bessel function of the first kind with $(\mathrm{m}=0,1,2, \ldots) . A_{m}$ and $B_{m}$ are unknown coefficients of field constants. Now, the equations for the electromagnetic fields for the metal cladding region having $\rho>R$ are as follows:

$E_{z}^{2}=C_{m} K_{m}\left(\gamma_{2} \rho\right) \exp (j m \theta) \exp (j \beta z)$

$E_{\varphi}^{2}=\frac{j}{\gamma_{2}}\left[\eta_{2} k_{m} D_{m} K_{m}^{\prime}\left(\gamma_{2} \rho\right)\right] \exp (j m \theta) \exp (j \beta z)$

$E_{\rho}^{2}=\frac{j}{\gamma_{2}}\left[\beta C_{m} K_{m}^{\prime}\left(\gamma_{2} \rho\right)\right] \exp (j m \theta) \exp (j \beta z)$

$H_{z}^{2}=D_{m} K_{m}\left(\gamma_{2} \rho\right) \exp (j m \theta) \exp (j \beta z)$

$H_{\varphi}^{2}=-\frac{j}{\eta_{2} \gamma_{2}}\left[k_{m} C_{m} K_{m}^{\prime}\left(\gamma_{2} \rho\right)\right] \exp (j m \theta) \exp (j \beta z)$

$H_{\rho}^{2}=\frac{j}{\gamma_{2}}\left[\beta D_{m} K_{m}^{\prime}\left(\gamma_{2} \rho\right)\right] \exp (j m \theta) \exp (j \beta z)$

In these equations, $\gamma_{2}=\sqrt{\left(\beta^{2}-k_{m}{ }^{2}\right)}$ represents the radial propagation constant, where $\beta$ is the wave vector, and $k_{2}^{2}=k_{o}^{2} \varepsilon_{m} \mu_{m}$ denotes the propagation constant of the metal region. $K_{m}\left(\gamma_{2} \rho\right)$ represents the modified Bessel function of the second kind where $(\mathrm{m}=0,1,2, \ldots) . C_{m}$ and $D_{m}$ are unknown coefficients, and $\eta_{2}=\sqrt{\frac{\mu_{m}}{\varepsilon_{m}}}$ is the impedance of the metal region.

The real part of wave vector $\beta$ (phase constant) is always greater than the wave vector of light, signifying that surface waves decay away from the graphene layer. However, the imaginary part exhibits the attenuation constant, indicating propagation loss in the $\mathrm{z}$ direction. The order of the SPP mode is governed by $\mathrm{m}$. The $m=0$ mode is the fundamental hybrid mode. For brevity, the phase factor $\exp (j \beta z+j m \theta-j \omega t)$ is neglected in the expressions of electromagnetic fields.

The plasmon modes are modeled at $\rho=\mathrm{R}$ employing the Kubo formalism due to the presence of graphene. The conductivity of graphene as a function of optical frequency $(\omega)$, chemical potential $\left(\mu_{\mathrm{c}}\right)$, scattering rate of carrier concentration $(\tau)$, and temperature $(\mathrm{T})$ [16] is comprised of two parts (i.e., interband and intraband conduction), as shown in the equation.

$\sigma_{g}\left(\omega, \mu_{c}, \tau, T\right)=j \frac{e^{2} K_{B} T}{\pi \hbar^{2}\left(\omega+\frac{j}{\tau}\right)}\left(\frac{\mu}{K_{B} T}+2 \log \left[e^{-\frac{\mu}{K_{B} T}}+1\right]\right)+j \frac{e^{2}}{4 \pi \hbar^{2}} \log \left[\frac{2|\mu|-\hbar\left(\omega+\frac{j}{\tau}\right)}{2|\mu|+\hbar\left(\omega+\frac{j}{\tau}\right)}\right]$

The modeling of the tangential components of the electromagnetic field on the CGM interface is obtained by using the impedance boundary conditions approach.

$E_{z}^{1}=E_{z}^{2}, E_{\varphi}^{1}=E_{\varphi}^{2}, H_{z}^{2}-H_{z}^{1}=-\sigma_{g} E_{\varphi}^{1}, \quad$ and $\quad H_{\varphi}^{2}-H_{\varphi}^{1}=\sigma_{g} E_{z}^{1}$

The dispersion relation for the hybrid surface plasmon mode is derived by employing the field equations in the boundary conditions, as given in Eq. 15:

$\frac{I_{0}^{\prime}\left(\gamma_{R} R\right)}{I_{0}\left(\gamma_{R} R\right)}\left\{\left[\frac{k_{m} K_{0}^{\prime}\left(\gamma_{2} R\right)}{\eta_{2} \gamma_{2} K_{0}\left(\gamma_{2} R\right)}+\frac{k_{-} I_{0}^{\prime}\left(\gamma_{L} R\right)}{\eta_{c} \sqrt{1+\chi^{2}} \gamma_{L} I_{0}\left(\gamma_{L} R\right)}+j \sigma_{g}\right]\left[\frac{\gamma_{2} k_{+} K_{0}\left(\gamma_{2} R\right)}{\eta_{2} k_{m} \gamma_{R} K_{0}^{\prime}\left(\gamma_{2} R\right)}-\frac{I_{0}\left(\gamma_{R} R\right)}{\eta_{c} \sqrt{1+\chi^{2}} I_{0}^{\prime}\left(\gamma_{R} R\right)}+\frac{j \sigma_{g} k_{+}}{\gamma_{R}}\right]\right\}+$ 


$$
\frac{I_{0}^{\prime}\left(\gamma_{L} R\right)}{I_{0}\left(\gamma_{L} R\right)}\left\{\left[\frac{k_{m} K_{0}^{\prime}\left(\gamma_{2} R\right)}{\eta_{2} \gamma_{2} K_{0}\left(\gamma_{2} R\right)}+\frac{k_{+} I_{0}^{\prime}\left(\gamma_{R} R\right)}{\eta_{c} \sqrt{1+\chi^{2}} \gamma_{R} I_{0}\left(\gamma_{R} R\right)}+j \sigma_{g}\right]\left[\frac{\gamma_{2} k_{-} K_{0}\left(\gamma_{2} R\right)}{\eta_{2} k_{m} \gamma_{L} K_{0}^{\prime}\left(\gamma_{2} R\right)}-\frac{I_{0}\left(\gamma_{L} R\right)}{\eta_{c} \sqrt{1+\chi^{2}} I_{0}^{\prime}\left(\gamma_{L} R\right)}+\frac{j \sigma_{g} k_{-}}{\gamma_{L}}\right]\right\}=0,
$$

where $K_{0}^{\prime}\left(\gamma_{2} R\right)=-K_{1}\left(\gamma_{2} R\right)$ and $I_{0}^{\prime}\left(\gamma_{R, L} R\right)=I_{1}\left(\gamma_{R, L} R\right)$ [19]. The first and third terms signify the dispersion relations for the TM waves. However, the dispersion relations associated with the TE waves at the interface between the CGM are presented in the second and fourth brackets. The above expression converges to the customary dispersion relation of SPPs supported by TM polarization at a dielectric-graphene-metal interface in the limit $\mathrm{k}_{+}=\mathrm{k}_{\text {-, }}$, when chirality becomes zero. The propagation characteristics of the hybrid plasmon modes along with the impact of various graphene parameters, chiral material parameters, and geometrical parameters on the propagative behavior of the surface waves are numerically calculated and presented in the next section.

\section{Results and Discussion}

The numerical simulations are executed using the Mathematica Wolfram 10.2 software package for the comprehensive understanding of the characteristics of the surface waves at the CGM interface of the proposed structure. Gold is considered a supporting metal for the proposed structure with a plasmon frequency of $\omega_{p}=1.30 \times 10^{16} \mathrm{rad} / \mathrm{s}$ and damping factor $\gamma=2.80 \times$ $10^{13} \mathrm{rad} / \mathrm{s}$ according to the Drude model [10]. For the understanding of the excitation and propagation of plasmon modes at the CGM interface, the dispersion relation is plotted between the normalized frequency $\left(\omega / \omega_{\mathrm{sp}}\right)$ and normalized propagation constant $\left(\beta / \mathrm{k}_{\mathrm{sp}}\right)$ in Fig. 2. The propagation constant is normalized by $k_{s p}=\frac{\omega_{s p}}{C}$ where $\omega_{s p}$ is the surface plasmon resonance frequency $\omega_{s p}=\frac{\omega_{p}}{\sqrt{1+n_{c}^{2}}}$ and $\mathrm{C}$ is the speed of light. The incident frequency $\omega$ is normalized by the surface plasmon resonance frequency $\omega_{s p}$. It is evident from Fig. 2 that the proposed geometry supports two types of modes corresponding to lower and higher frequency values. These modes emerge for higher values of chirality, and their band gap also exhibits chiral dependence. The rise in the chiral strength reduces the band gap between higher and lower modes. The lower mode appears to be slow moving, while higher order modes are quite fast. The generation of higher modes for certain chiral values indicates the existence of hybrid plasmon modes that decouple for greater chiral strength $\left(\xi=0.008 \Omega^{-1}\right.$ and $0.02 \Omega^{-1}$ ), enabling the opportunity of chiral sensing for the proposed structure. This behavior of surface waves is the opposite of that of the plasmon modes presented for a CGM planar structure in [16]. The dispersion curve plotted between the incident frequency $\omega$ and propagation constant $\beta / k_{0}$ under special conditions (i.e., $\xi=0.0 \Omega^{-1}$ and permittivity of cladding region as $\varepsilon_{\mathrm{m}}=1.2 \varepsilon_{\mathrm{o}}$ ) converges to only a single TM plasmon mode, which is quite similar to the surface plasmon modes propagating in the dielectric-filled graphene-coated cylindrical waveguide embedded in a dielectric environment, as presented in Fig. 3 [19]. This confirms the authenticity and accuracy of the numerical simulation generated in the present work. This also ensures that the proposed structure is supporting hybrid plasmon modes, as chiral strength is responsible for the decoupling of the TE-TM plasmon modes. The squeezing of the band gap with the increasing value of the chiral strength also unveils another degree of freedom for the modulation of the surface waves. 


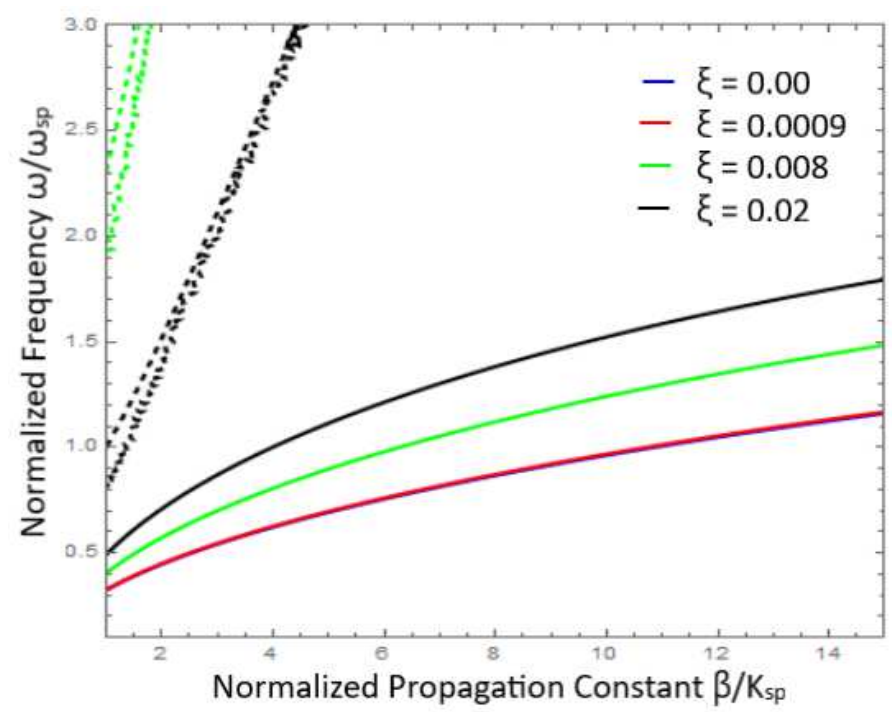

Fig. 2. Dispersion curve of surface waves at CGM interface as function of chiral strength with $\mathrm{T}$ $=300 \mathrm{~K}, \tau=1.6 \mathrm{ps}$, and $\mu_{\mathrm{c}}=0.6 \mathrm{eV}$.

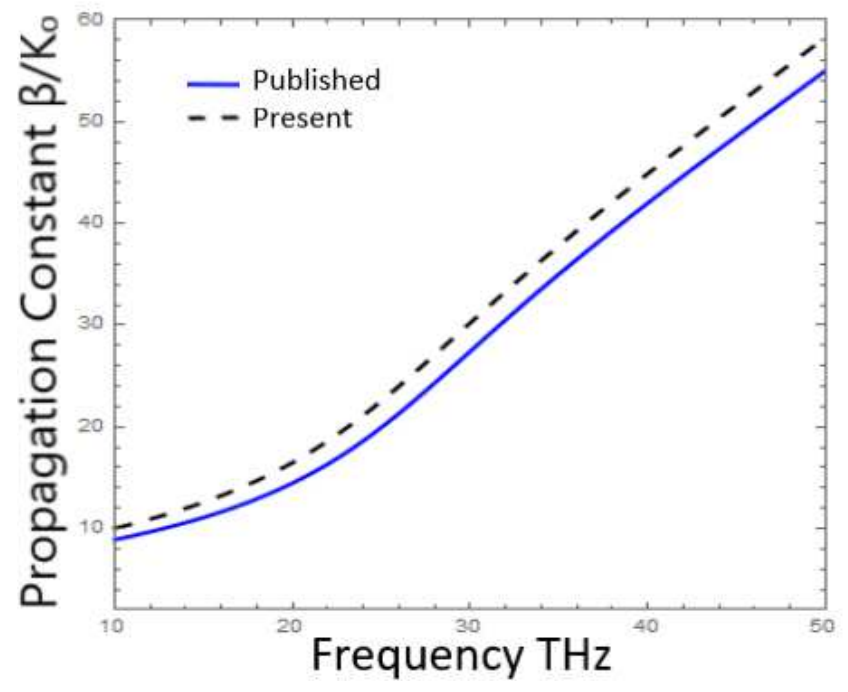

Fig. 3. Comparison of dispersion curve for hybrid surface waves at CGM interface with $\xi_{\mathrm{c}}=0.0 \Omega^{-}$ ${ }^{1}$ and dispersion curve of fundamental plasmon mode at dielectric-graphene-dielectric interface [19].

The physical existence of the hybrid surface wave excitation and propagation at the CGM interface can also be confirmed by plotting the curve between the normalized field distribution $\mathrm{E}_{\mathrm{z}}$ and radial distance R, as shown in Fig. 4a \& b. Fig. 4a represents the exponential growth, while Fig. 4b indicates the exponential decay of plasmon modes propagating away from the interface, which is a key feature of surface waves [20]. 

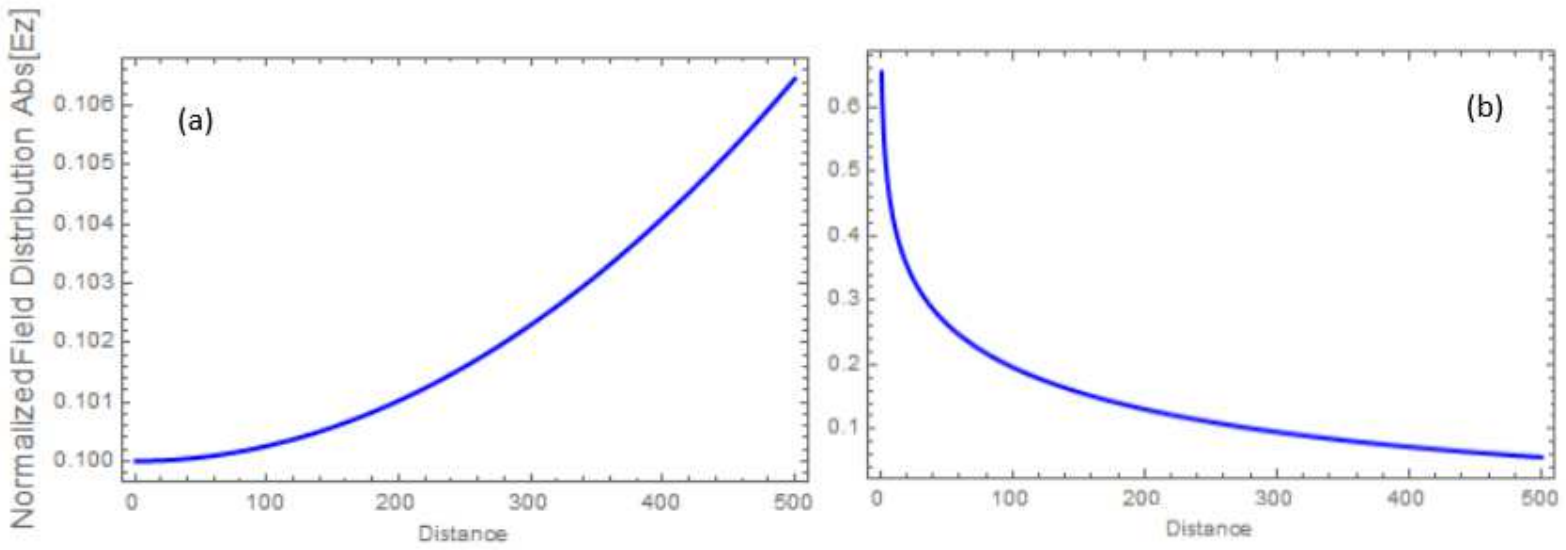

Fig. 4. Normalized field distribution of chiral dielectric and metal cladding region as function of radial distance with $\mathrm{T}=300 \mathrm{k}, \tau=1.6 \mathrm{ps}$, and $\mu_{\mathrm{c}}=0.6 \mathrm{eV}$ for CGM with $\xi=0.003 \Omega^{-1}$.

The presence of the graphene layer offers an opportunity for the active and dynamical tuning of the surface waves by altering the fermi level/chemical potential or chemical doping of graphene. For this purpose, a plot of the dispersion curve between the normalized propagation constant and normalized frequency for various values of chemical potential $\left(\mu_{\mathrm{c}}=0.2 \mathrm{eV}, \mu_{\mathrm{c}}=0.6 \mathrm{eV}, \mu_{\mathrm{c}}=1.0\right.$ $\mathrm{eV}$, and $\mu_{\mathrm{c}}=1.5 \mathrm{eV}$ ) is presented in Fig. 5. The chiral strength and radius of the waveguide are considered as $\xi=0.008 \Omega^{-1}$ and $\mathrm{R}=100 \mathrm{~nm}$, respectively. The results reveal a significant role of the chemical potential in the modulation of the lower modes of surface plasmons, as indicated by the variation or rise in plasmon resonance frequencies by increasing the gating voltage of graphene. However, higher modes exhibit insensitive behavior for any change in the chemical potential of graphene, which is evident by the overlapping of the curves of higher plasmon modes. The influence of the chemical potential of graphene is modeled on the propagation of the hybrid plasmon modes in the $(0.1-10 \mathrm{THz})$ spectral range due to the special properties of graphene in the $\mathrm{THz}$ frequency regime. Fig. 6. describes a plot between the incident frequency $\omega$ in $\mathrm{THz}$ and normalized propagation constant $\beta / \mathrm{k}_{\mathrm{sp}}$ as a function of the chemical potential of graphene, which describes nonlinear behavior for both plasmon modes. The propagation constant of the lower mode of hybrid surface waves is significantly altered by a rise in the gating voltage of graphene. The decrease in the propagation constant depicts a loosely confined field for a larger biasing voltage. However, higher plasmon modes express negligible variation in the propagation constant by varying the chemical potential. Although the propagation constant is reduced when applied with strong biasing values, this change is not significant for higher modes. 


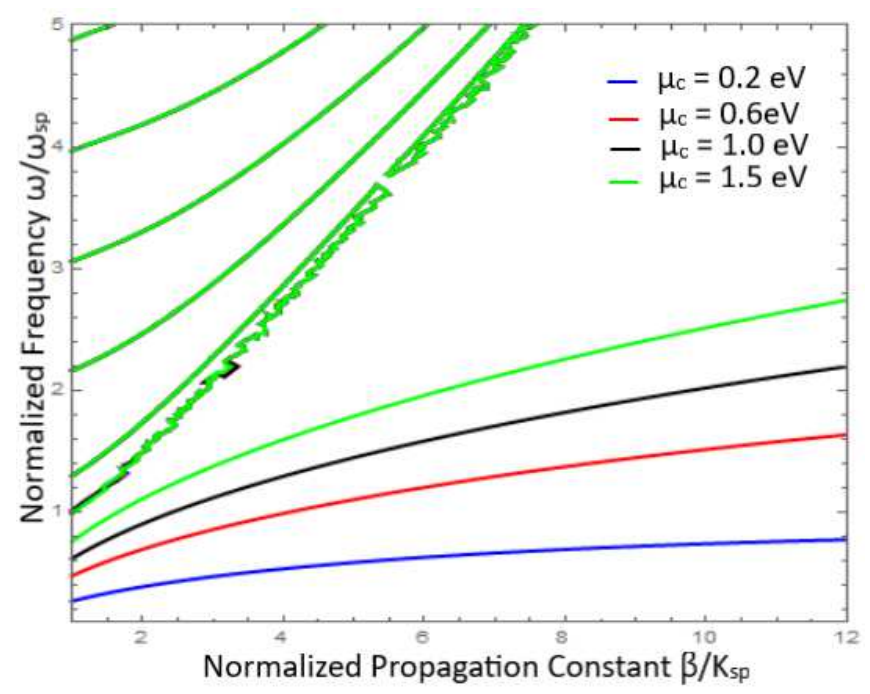

Fig. 5. Dispersion curve of hybrid surface waves at CGM interface as function of chemical potential with $\mathrm{T}=300 \mathrm{~K}, \tau=1.6 \mathrm{ps}$, and $\xi_{\mathrm{c}}=0.008 \Omega^{-1}$.

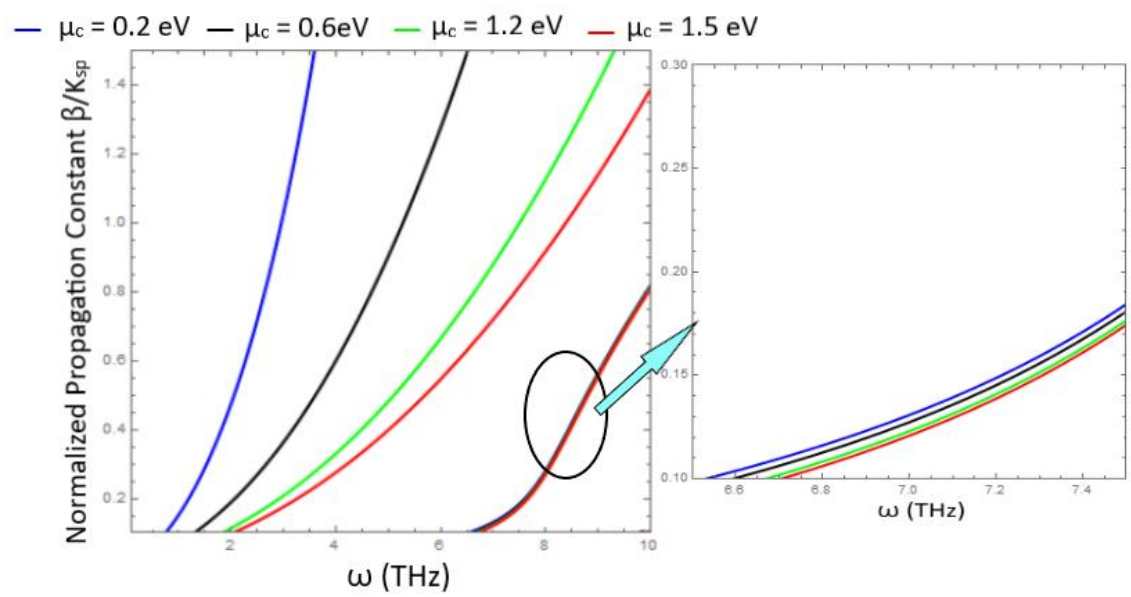

Fig. 6. Effect of chemical potential on normalized propagation constant as function of incident frequency with $\mathrm{T}=300 \mathrm{~K}, \tau=1.6 \mathrm{ps}$, and $\xi_{\mathrm{c}}=0.02 \Omega^{-1}$.

The combined effect of chiral strength and chemical potential on the propagation of the surface waves is presented in Fig. 7. The chemical potential values are considered as $\left(\mu_{\mathrm{c}}=0.1 \mathrm{eV}, \mu_{\mathrm{c}}=0.2\right.$ $\mathrm{eV}, \mu_{\mathrm{c}}=0.3 \mathrm{eV}$, and $\left.\mu_{\mathrm{c}}=0.4 \mathrm{eV}\right)$ with their corresponding chiral strength values $\left(\xi=0.01 \Omega^{-1}, \xi=\right.$ $0.02 \Omega^{-1}, \xi=0.03 \Omega^{-1}$, and $\xi=0.04 \Omega^{-1}$ ). The convergence of the forbidden region is observed for higher values of chemical potential and chiral strength. The breaking of symmetry of the plasmon mode at $0.04 \Omega^{-1}$ indicates the decoupling of TE-TM plasmon modes due to the Goos-Hänchen shift. The increase in the number of harmonics is also witnessed for greater chirality [21]. Keeping in mind the characteristics of surface waves (i.e., decaying away from the interface), the influence of radial distance on the propagation of the hybrid surface waves at the CGM interface is depicted in Fig. 8. 


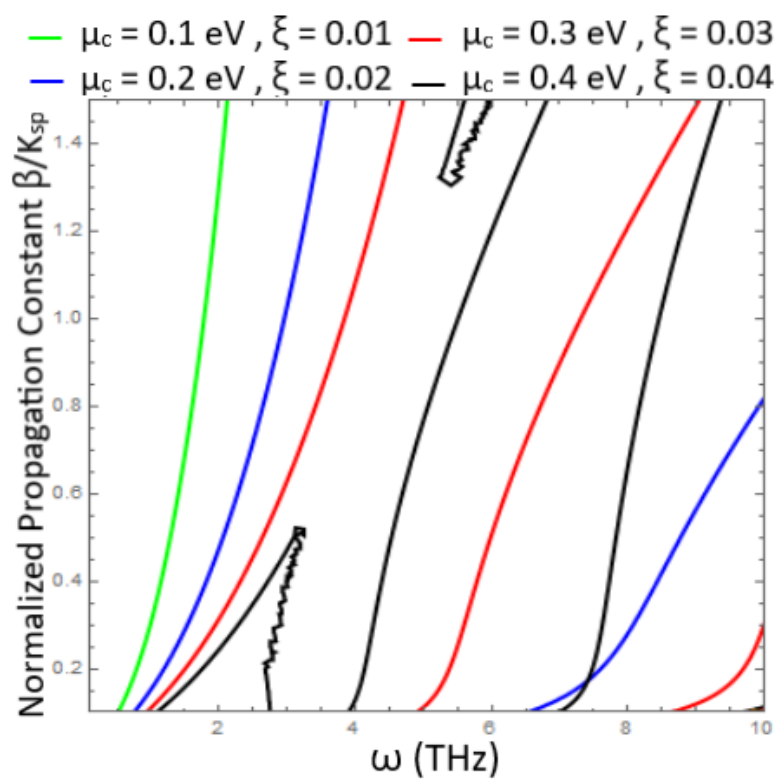

Fig. 7. Combined effect of chiral strength and chemical potential on normalized propagation constant of hybrid surface waves at CGM interface with $\mathrm{T}=300 \mathrm{~K}$ and $\tau=1.6 \mathrm{ps}$.

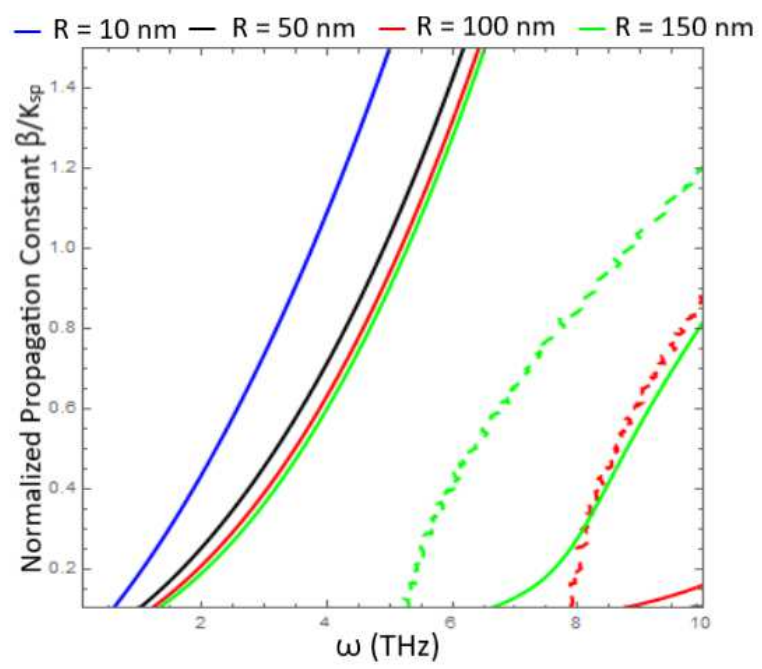

Fig. 8. Effect of radius on normalized propagation constant of hybrid surface wave at CGM interface with $\mathrm{T}=300 \mathrm{~K}, \tau=1.6 \mathrm{ps}, \xi_{\mathrm{c}}=0.02 \Omega^{-1}$, and $\mu_{\mathrm{c}}=0.6 \mathrm{eV}$.

The dispersion curve is plotted between the incident frequency $\omega$ and normalized propagation constant for various values of radius (i.e., $R=10 \mathrm{~nm}, \mathrm{R}=50 \mathrm{~nm}, \mathrm{R}=100 \mathrm{~nm}$, and $\mathrm{R}=150 \mathrm{~nm}$ ), and it is observed that for thin cylindrical waveguides with small diameter, only a single mode propagates at the CGM interface. The splitting of the mode occurs for thick cores. The lower and higher symmetric plasmon modes along with antisymmetric plasmon modes originate by increasing the radius of the core. However, the lower mode exhibits a negligible reduction in field confinement for a thick core as compared to a significant increase in the propagation constant for higher modes with an increase in the core radius. The convergence of the forbidden region by increasing the radius reveals the tuning of the band gap as a function of radius [15]. The single 
plasmon mode displays a significant reduction in field confinement when the radial distance of the waveguide is increased.

The effective refractive index as a function of normalized frequency is plotted against different chiral strength values (i.e., $\xi=0.008 \Omega^{-1}, \xi=0.009 \Omega^{-1}, \xi=0.02 \Omega^{-1}, \xi=0.03 \Omega^{-1}$ ) and $\mu_{\mathrm{c}}=0.6 \mathrm{eV}$ and presented in Fig. 9. The cutoff effect arises at a normalized frequency of 0.46 . For smaller values of chirality, the plasmons modes exhibit no significant difference for refractive index values up to 1.0. However, for cutoff-free higher refractive indices, significant variation occurs in the propagation behavior of surface waves. The rise in the chiral strength promotes the Goos-Hänchen effect, and backward directing higher modes appear for refractive index values greater than 1.5.

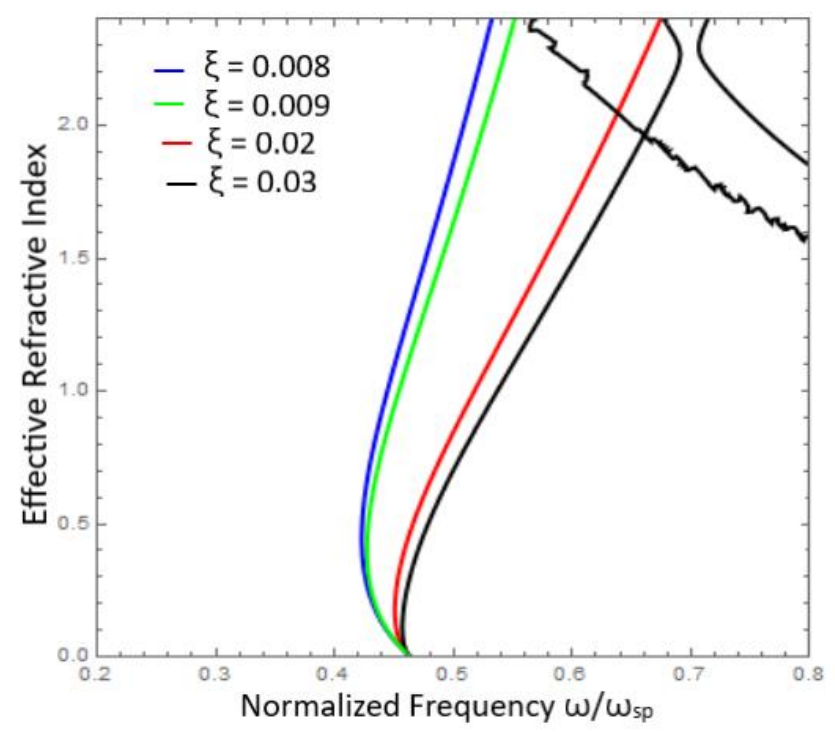

Fig. 9. Effect of chiral strength on effective refractive index as function of normalized frequency with $\mathrm{T}=300 \mathrm{~K}, \tau=1.6 \mathrm{ps}$, and $\mu_{\mathrm{c}}=0.6 \mathrm{eV}$.

Two of the main goals of graphene plasmonics are to reduce the propagation loss and to enhance the propagation length of plasmon modes. The propagation length $L_{p}=\frac{1}{2 \operatorname{Im}[\beta]}$ depends on the imaginary part of the propagation constant. Fig. 10 presents a plot between the propagation length and incident frequency as a function of chiral strength and the chemical potential of graphene. The chiral strength values are considered as $\xi=0.006 \Omega^{-1}, \xi=0.009 \Omega^{-1}, \xi=0.01 \Omega^{-1}$, and $\xi=0.02 \Omega^{-}$ ${ }^{1}$ with their corresponding chemical potential values of $\mu_{\mathrm{c}}=0.6 \mathrm{eV}, \mu_{\mathrm{c}}=0.9 \mathrm{eV}, \mu_{\mathrm{c}}=1.0 \mathrm{eV}$, and $\mu_{\mathrm{c}}=2.0 \mathrm{eV}$. The results reveal the modulation of propagation length by varying the biasing voltage of graphene and chiral strength. The propagation length increases linearly by increasing the incident frequency. The propagation length significantly increases at higher frequencies for larger values of chemical potential and chiral strength. The splitting of plasmon modes is also observed for $\xi=0.01 \Omega^{-1}, \mu_{\mathrm{c}}=1.0 \mathrm{eV}$ and $\xi=0.02 \Omega^{-1}, \mu_{\mathrm{c}}=2.0 \mathrm{eV}$ with higher decoupled modes. A sudden upsurge in propagation length approaching $10 \mu \mathrm{m}$ is noticed for $50-85 \mathrm{THz}$ for $\xi=0.02 \Omega^{-1}, \mu_{\mathrm{c}}=$ $2.0 \mathrm{eV}$. 


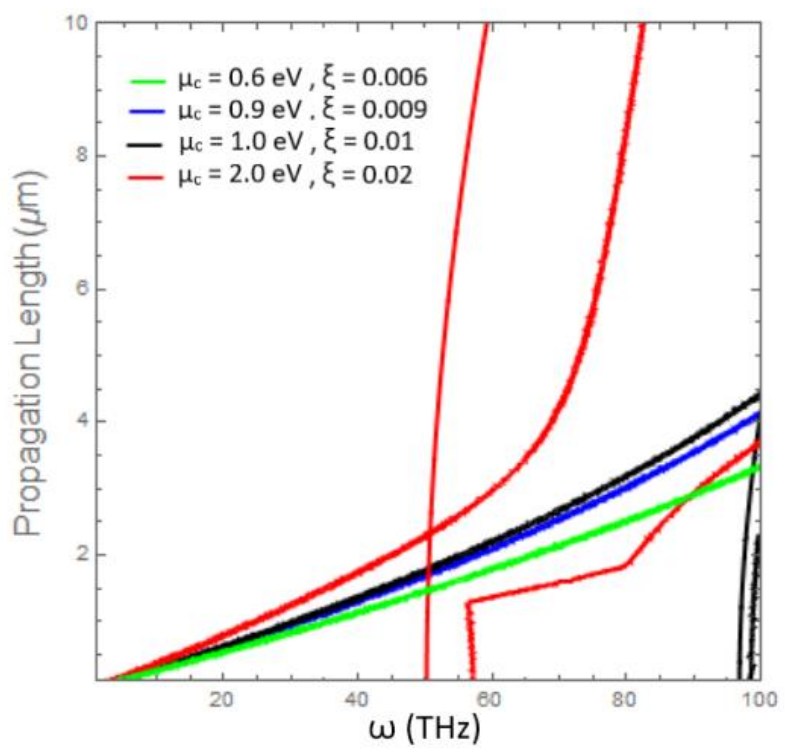

Fig. 10. Effect of chiral strength and chemical potential on propagation length of hybrid plasmon modes at CGM interface.

The relation of cutoff chiral values $\left(\xi_{\mathrm{c}}\right)$ is plotted as a function of normalized frequency against various values of refractive index (i.e., $\mathrm{n}_{\mathrm{c}}=1.3,1.35,1.40$ ) in Fig. 11. The cutoff condition is obtained as depicted by $[10,16]$ (i.e., by considering $\mathrm{n}_{+}>\mathrm{n}_{\text {- }}$ and $\beta=\mathrm{k}_{+}$providing $\gamma_{R}=0$ and $\gamma_{L}=\left(k_{+}^{2}-k_{-}^{2}\right)^{\frac{1}{2}}$ in Eq. 15). The graph exhibits the sensitive behavior of plasmon modes toward optical and chemical changes in the chiral medium. The cutoff chiral values follow a linear and distinct relation for variation in refractive index values within a narrow frequency range. The frequency-dependent cutoff values for chiral media having different refractive indices enrich the sensitivity of the proposed design toward chiroptical and chemical sensing.

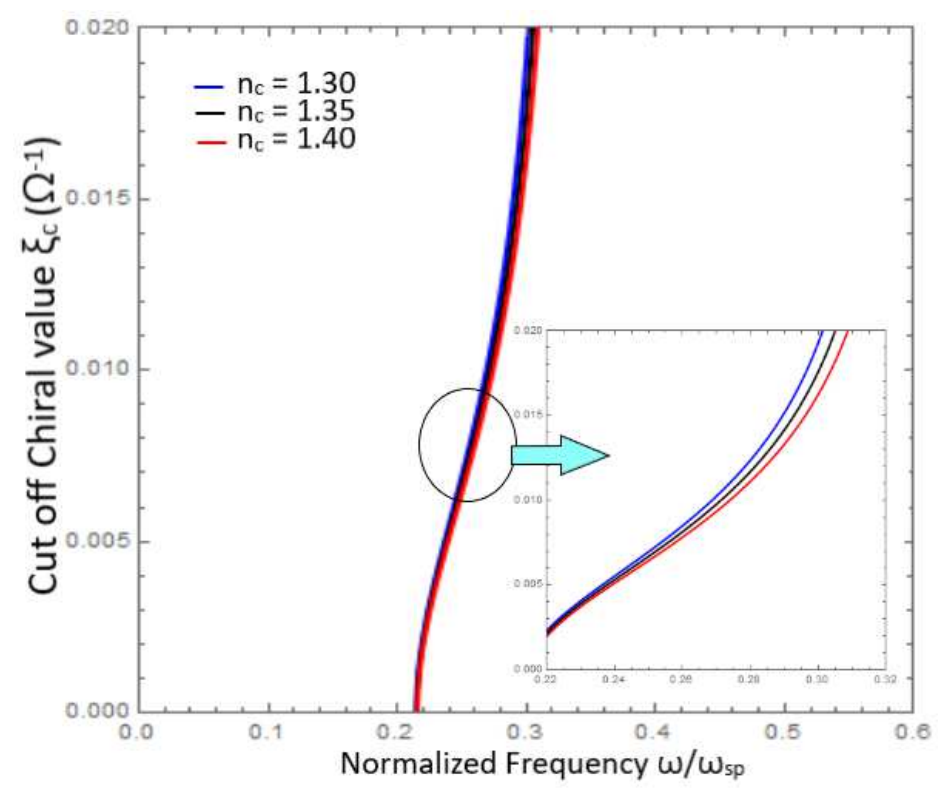


Fig. 11. Effect of refractive indices on cutoff chiral strength as function of normalized frequency with $\mathrm{T}=300 \mathrm{~K}, \tau=1.6 \mathrm{ps}$, and $\mu_{\mathrm{c}}=0.2 \mathrm{eV}$.

\section{Conclusion}

For chiroptical and chemical sensing, a chiral-filled graphene-based metal-coated cylindrical waveguide was presented. The excitation and propagation of hybrid plasmon modes was studied at the CGM interface. The dispersion relation for the proposed structure was derived by obtaining the analytical solution of Maxwell's equations using impedance matching boundary conditions, and the following conclusions were drawn:

i. Hybrid surface plasmon modes consisting of lower and higher modes propagate along the CGM interface.

ii. The frequency band gap between the lower and higher plasmon modes can be tuned by altering the chirality and chemical potential of graphene and the radial distance of the cylindrical waveguide.

iii. The normalized propagation constant of the lower plasmon modes is highly sensitive to the chemical potential of graphene. However, the chemical potential has no significant effect on higher plasmon modes.

iv. The decoupling of plasmon modes representing the Goos-Hänchen effect is noticed for higher values of chirality.

v. The effective refractive index and propagation length of surface plasmon modes can be modulated by varying the chiral strength and chemical potential of graphene. A high propagation length is achieved for greater chiral strength in a high frequency regime.

vi. The proposed structure may have potential applications for chiroptical and chemical sensing and enantiomeric detection in the $\mathrm{THz}$ frequency regime.

Acknowledgements: The authors would like to extend their sincere appreciation to the Deanship of Scientific Research (DSR) at King Saud University, Riyadh, Saudi Arabia for their financial support through the Research Group Project No. RG-1436-01.

\section{References}

[1] Y. Liu, X. Dong, and P. Chen, "Biological and chemical sensors based on graphene materials," Chemical Society Reviews, vol. 41, pp. 2283-2307, 2012.

[2] J. T. Collins, C. Kuppe, D. C. Hooper, C. Sibilia, M. Centini, and V. K. Valev, "Chirality and chiroptical effects in metal nanostructures: fundamentals and current trends," Advanced Optical Materials, vol. 5, p. 1700182, 2017.

[3] S. A. Maier, Plasmonics: fundamentals and applications: Springer Science \& Business Media, 2007.

[4] W. Guo, L. Wang, X. Chen, C. Liu, W. Tang, C. Guo, et al., "Graphene-based broadband terahertz detector integrated with a square-spiral antenna," Optics Letters, vol. 43, pp. 1647-1650, 2018. 
[5] C.-H. Liu, Y.-C. Chang, T. B. Norris, and Z. Zhong, "Graphene photodetectors with ultrabroadband and high responsivity at room temperature," Nature Nanotechnology, vol. 9, pp. 273-278, 2014.

[6] M. Liu, X. Yin, and X. Zhang, "Double-layer graphene optical modulator," Nano Letters, vol. 12, pp. 1482-1485, 2012.

[7] O. Krupin, H. Asiri, C. Wang, R. N. Tait, and P. Berini, "Biosensing using straight longrange surface plasmon waveguides," Optics Express, vol. 21, pp. 698-709, 2013.

[8] B. M. Maoz, Y. Chaikin, A. B. Tesler, O. Bar Elli, Z. Fan, A. O. Govorov, et al., "Amplification of chiroptical activity of chiral biomolecules by surface plasmons," Nano Letters, vol. 13, pp. 1203-1209, 2013.

[9] A. Ghaffar and M. A. S. Alkanhal, "Electromagnetic waves in parallel plate uniaxial anisotropic chiral waveguides," Optical Materials Express, vol. 4, pp. 1756-1761, 2014.

[10] G. Mi and V. Van, "Characteristics of surface plasmon polaritons at a chiral-metal interface," Optics Letters, vol. 39, pp. 2028-2031, 2014.

[11] M. Z. Yaqoob, A. Ghaffar, M. Alkanhal, S. U. Rehman, and F. Razzaz, "Hybrid Surface plasmon polariton wave generation and modulation by chiral-graphene-metal (CGM) structure," Scientific Reports, vol. 8, p. 18029, 2018.

[12] I. Toqeer, A. Ghaffar, M. Y. Naz, and B. Sultana, "Characteristics of dispersion modes supported by graphene chiral graphene waveguide," Optik, vol. 186, pp. 28-33, 2019.

[13] V. Kesari and J. Keshari, "Hybrid-mode analysis of circular waveguide with chiral dielectric lining for dispersion characteristics for potential application in broadbanding a gyro-traveling-wave tube," Journal of Electromagnetic Waves and Applications, vol. 33, pp. 204-214, 2019.

[14] J.-F. Dong and J. Li, "Characteristics of guided modes in uniaxial chiral circular waveguides," Progress in Electromagnetics Research, vol. 124, pp. 331-345, 2012.

[15] Q. Zhang, J. Li, X. Liu, and D. J. Gelmecha, "Dispersion, propagation, and transverse spin of surface plasmon polaritons in a metal-chiral-metal waveguide," Applied Physics Letters, vol. 110, p. 161114, 2017.

[16] M. Yaqoob, A. Ghaffar, M. Alkanhal, S. U. Rehman, and F. Razzaz, "Hybrid surface plasmon polariton wave generation and modulation by chiral-graphene-metal (CGM) structure," Scientific Reports, vol. 8, pp. 1-9, 2018.

[17] S. E. Swiontek, D. P. Pulsifer, and A. Lakhtakia, "Optical sensing of analytes in aqueous solutions with a multiple surface-plasmon-polariton-wave platform," Scientific Reports, vol. 3, pp. 1-6, 2013.

[18] F. Abbas, A. Lakhtakia, Q. A. Naqvi, and M. Faryad, "An optical-sensing modality that exploits Dyakonov-Tamm waves," Photonics Research, vol. 3, pp. 5-8, 2015.

[19] Y. Gao, G. Ren, B. Zhu, H. Liu, Y. Lian, and S. Jian, "Analytical model for plasmon modes in graphene-coated nanowire," Optics Express, vol. 22, pp. 24322-24331, 2014.

[20] M. Ali, A. Ghaffar, M. A. Alkanhal, and Y. Khan, "Study of hybrid surface plasmon modes in metallic circular waveguide filled with magnetized plasma," Waves in Random and Complex Media, pp. 1-14, 2020.

[21] R. Zhou, S. Yang, D. Liu, and G. Cao, "Confined surface plasmon of fundamental wave and second harmonic waves in graphene nanoribbon arrays," Optics Express, vol. 25, pp. 31478-31491, 2017. 


\section{Figures}

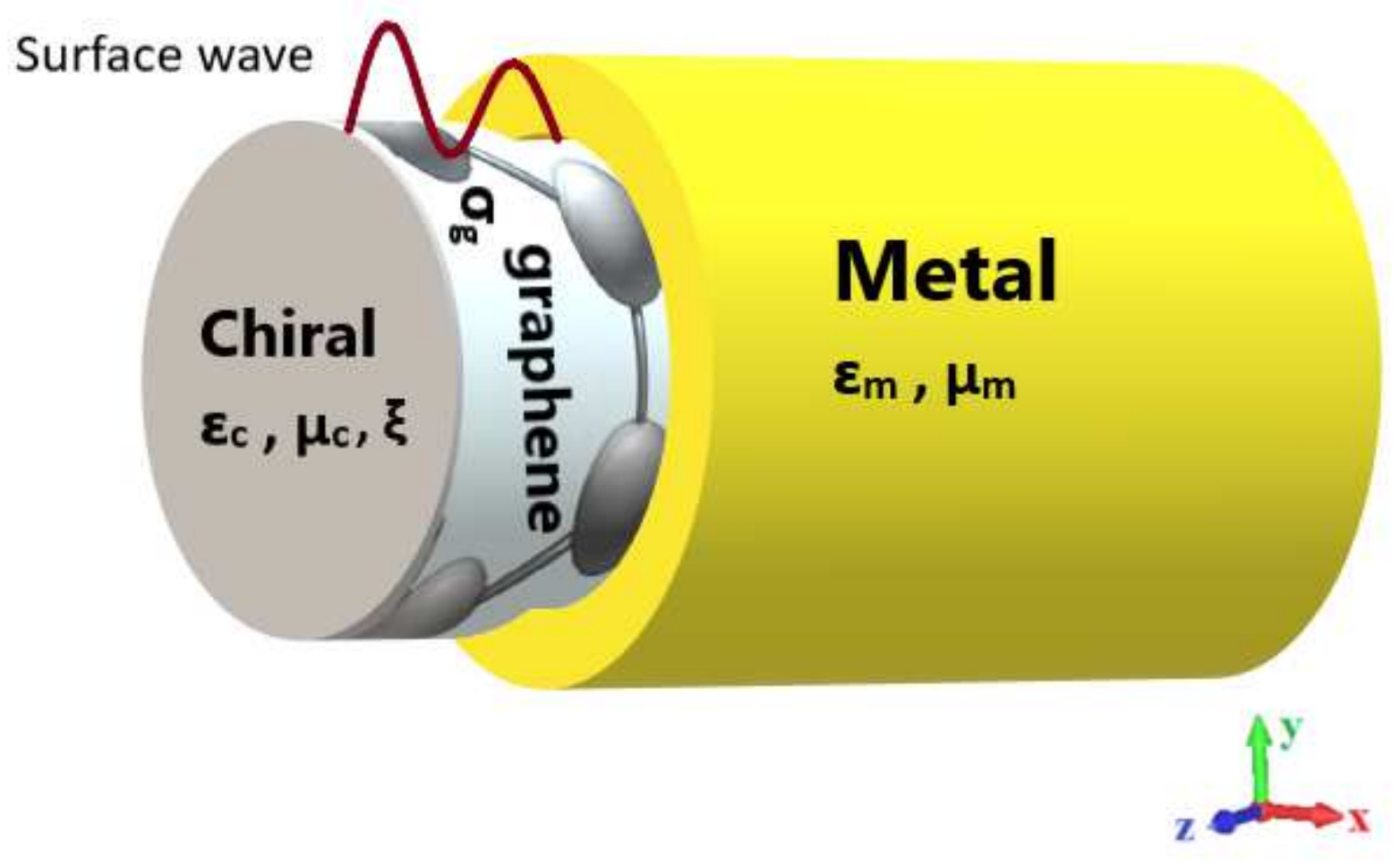

Figure 1

Surface wave excitation and propagation in CGM-based cylindrical structure 


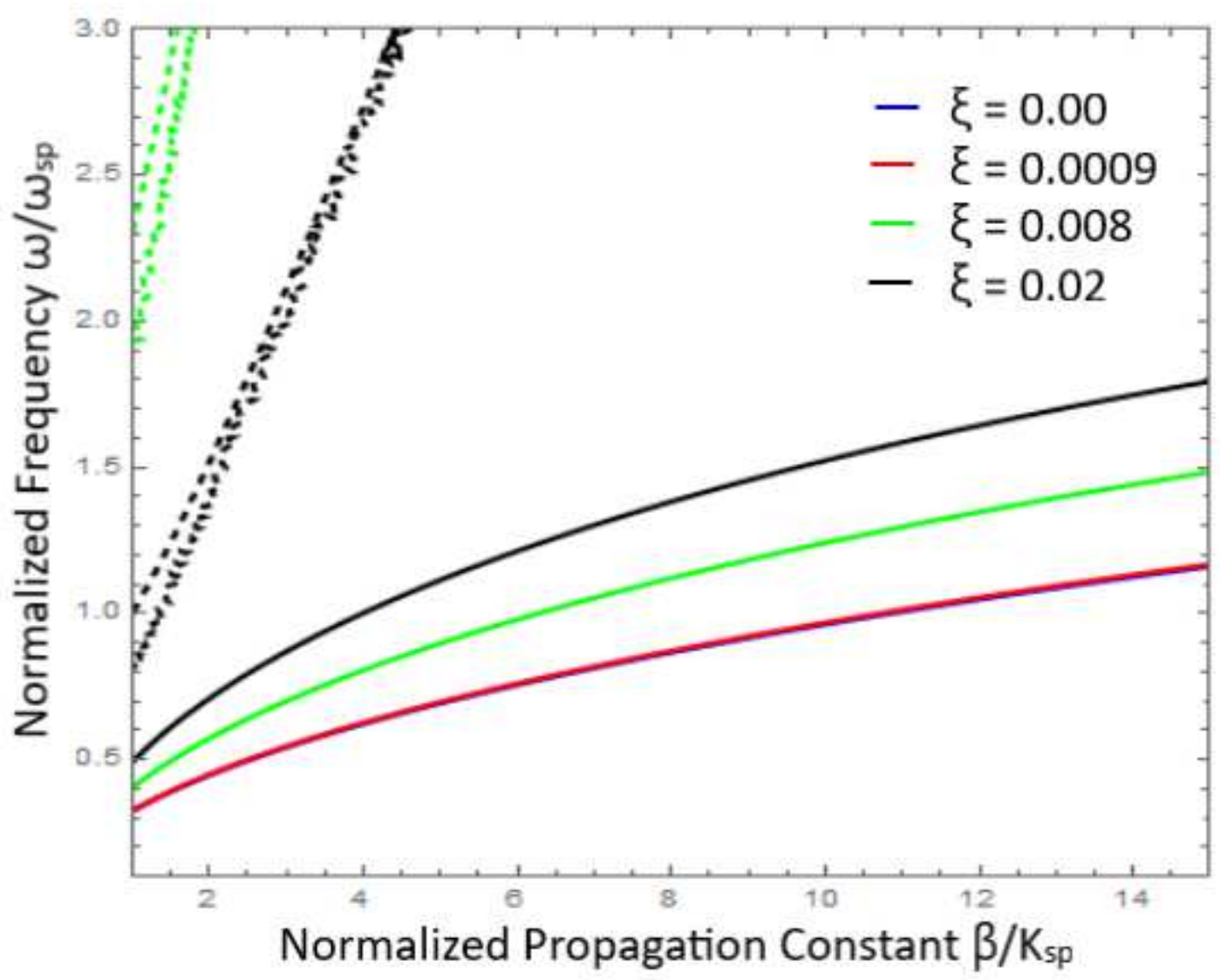

Figure 2

Dispersion curve of surface waves at CGM interface as function of chiral strength with $T=300 \mathrm{~K}, \tau=$ $1.6 \mathrm{ps}$, and $\mu \mathrm{c}=0.6 \mathrm{eV}$.

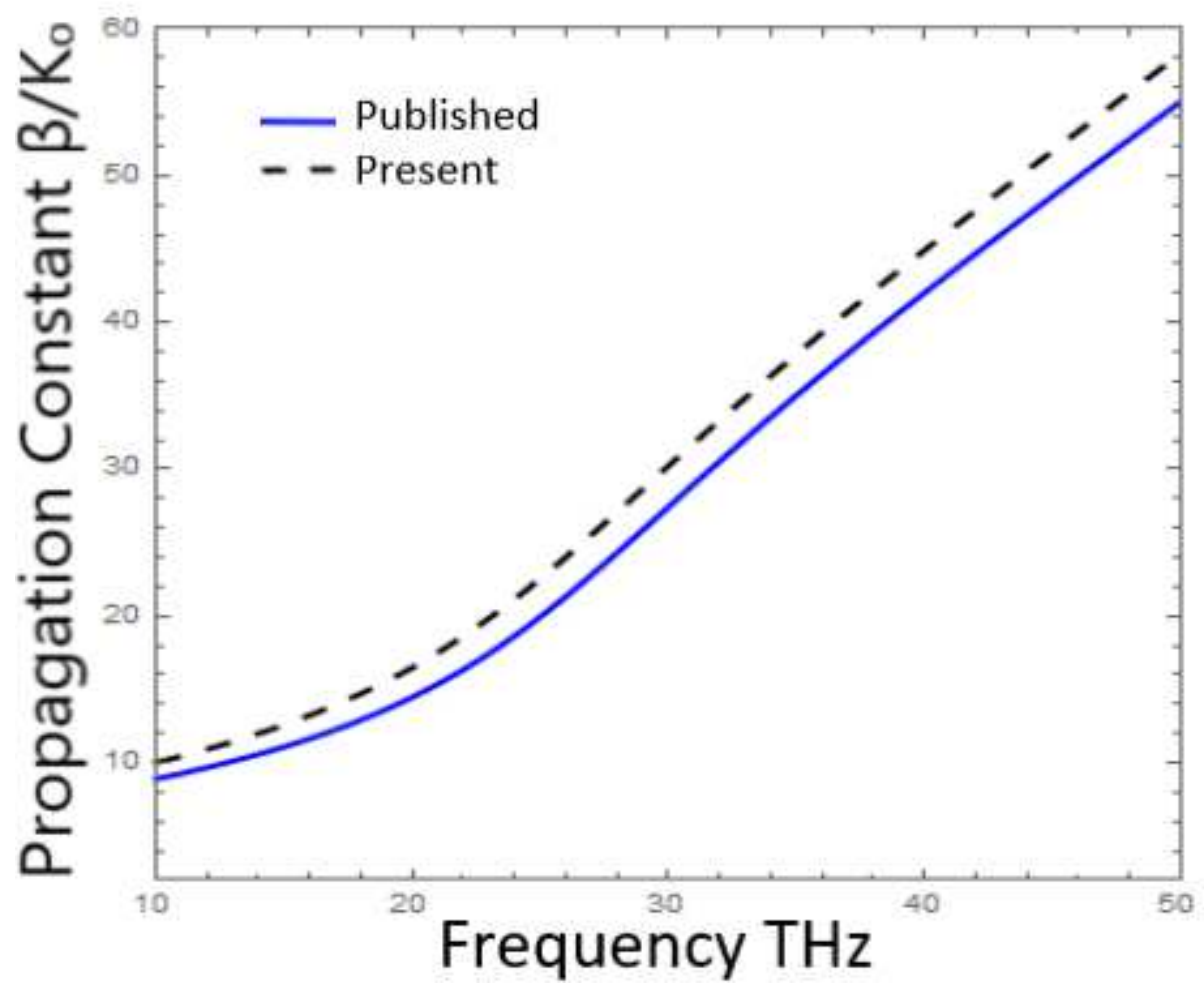




\section{Figure 3}

Comparison of dispersion curve for hybrid surface waves at CGM interface with $\xi c=0.0 \Omega-1$ and dispersion curve of fundamental plasmon mode at dielectric-graphene-dielectric interface [19].
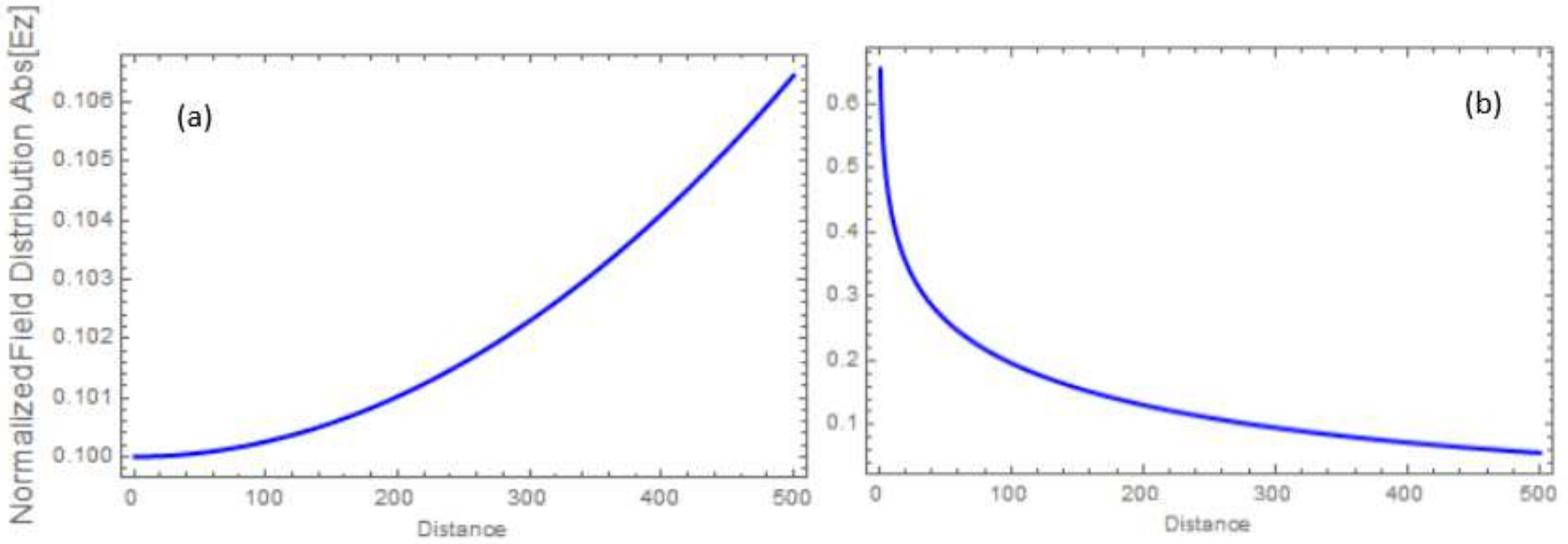

\section{Figure 4}

Normalized field distribution of chiral dielectric and metal cladding region as function of radial distance with $\mathrm{T}=300 \mathrm{k}, \mathbb{Q}=1.6 \mathrm{ps}$, and $\mu \mathrm{c}=0.6 \mathrm{eV}$ for CGM with $\xi=0.003 \Omega-1$.

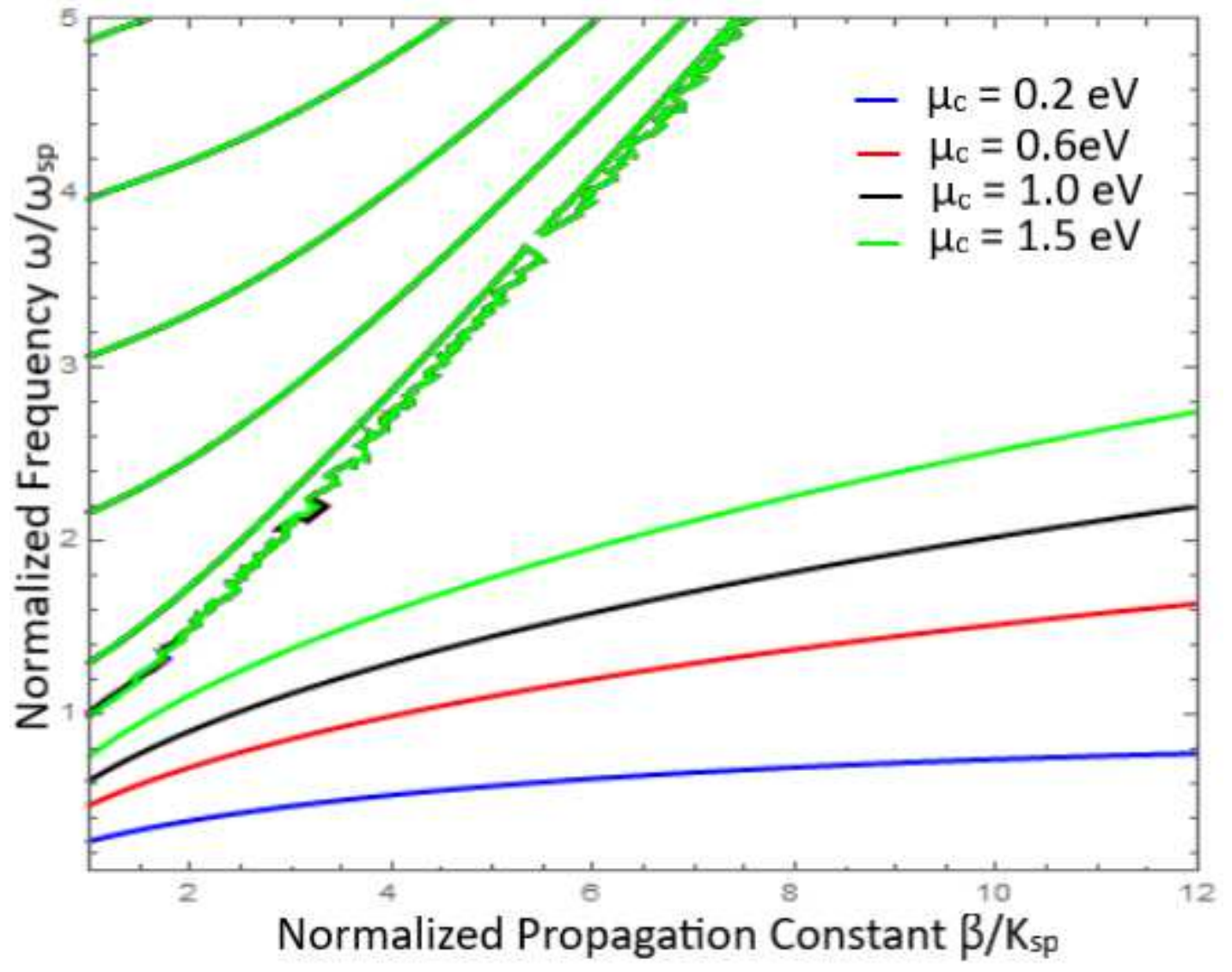

Figure 5 
Dispersion curve of hybrid surface waves at CGM interface as function of chemical potential with $\mathrm{T}=$ $300 \mathrm{~K}, \tau=1.6 \mathrm{ps}$, and $\xi \mathrm{c}=0.008 \Omega-1$.

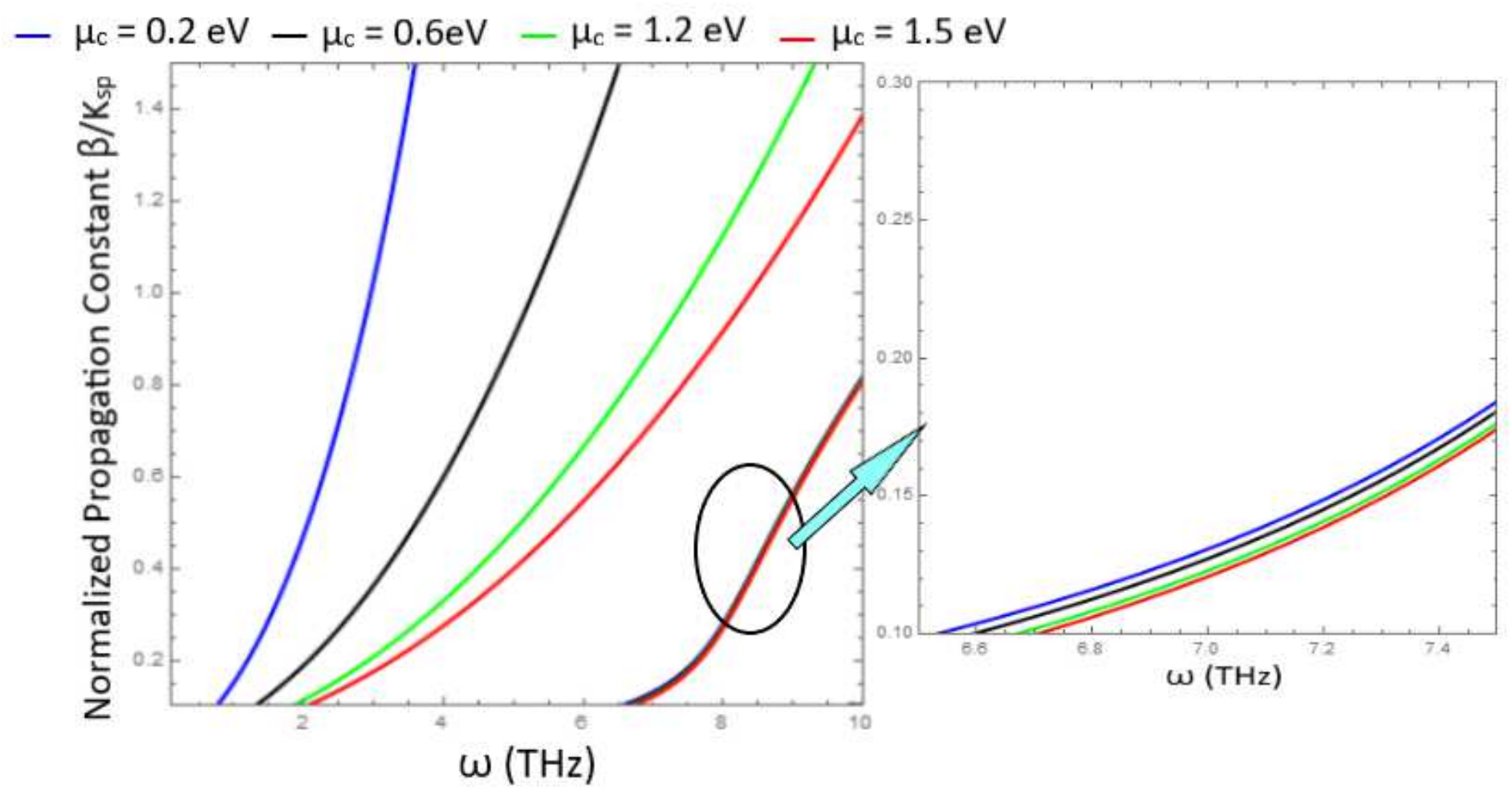

Figure 6

Effect of chemical potential on normalized propagation constant as function of incident frequency with $T$ $=300 \mathrm{~K}, \tau=1.6 \mathrm{ps}$, and $\xi \mathrm{c}=0.02 \Omega-1$. 


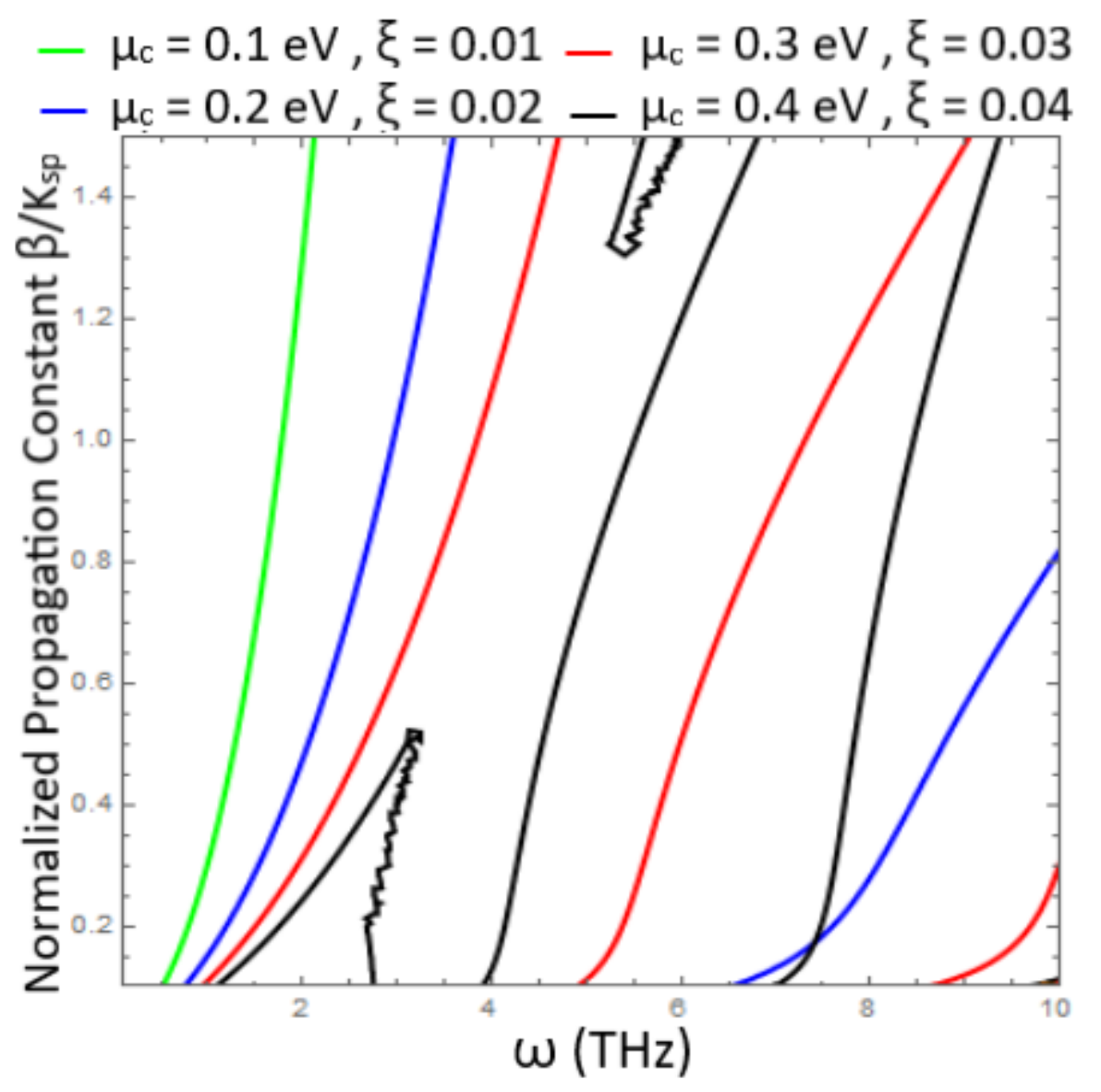

Figure 7

Combined effect of chiral strength and chemical potential on normalized propagation constant of hybrid surface waves at CGM interface with $\mathrm{T}=300 \mathrm{~K}$ and $\tau=1.6 \mathrm{ps}$. 


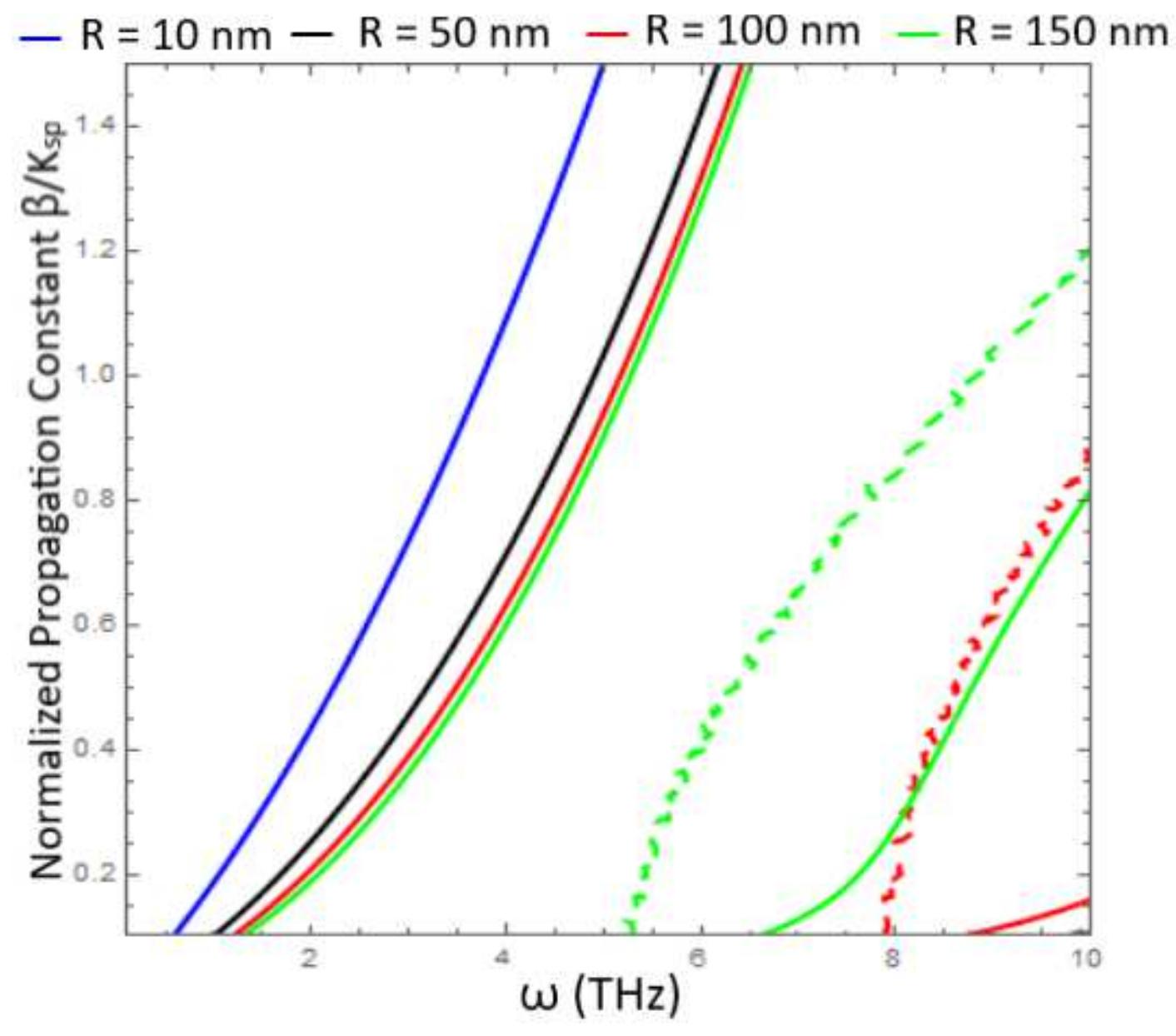

Figure 8

Effect of radius on normalized propagation constant of hybrid surface wave at CGM interface with $\mathrm{T}=$ $300 \mathrm{~K}, \tau=1.6 \mathrm{ps}, \xi \mathrm{c}=0.02 \Omega-1$, and $\mu \mathrm{c}=0.6 \mathrm{eV}$. 


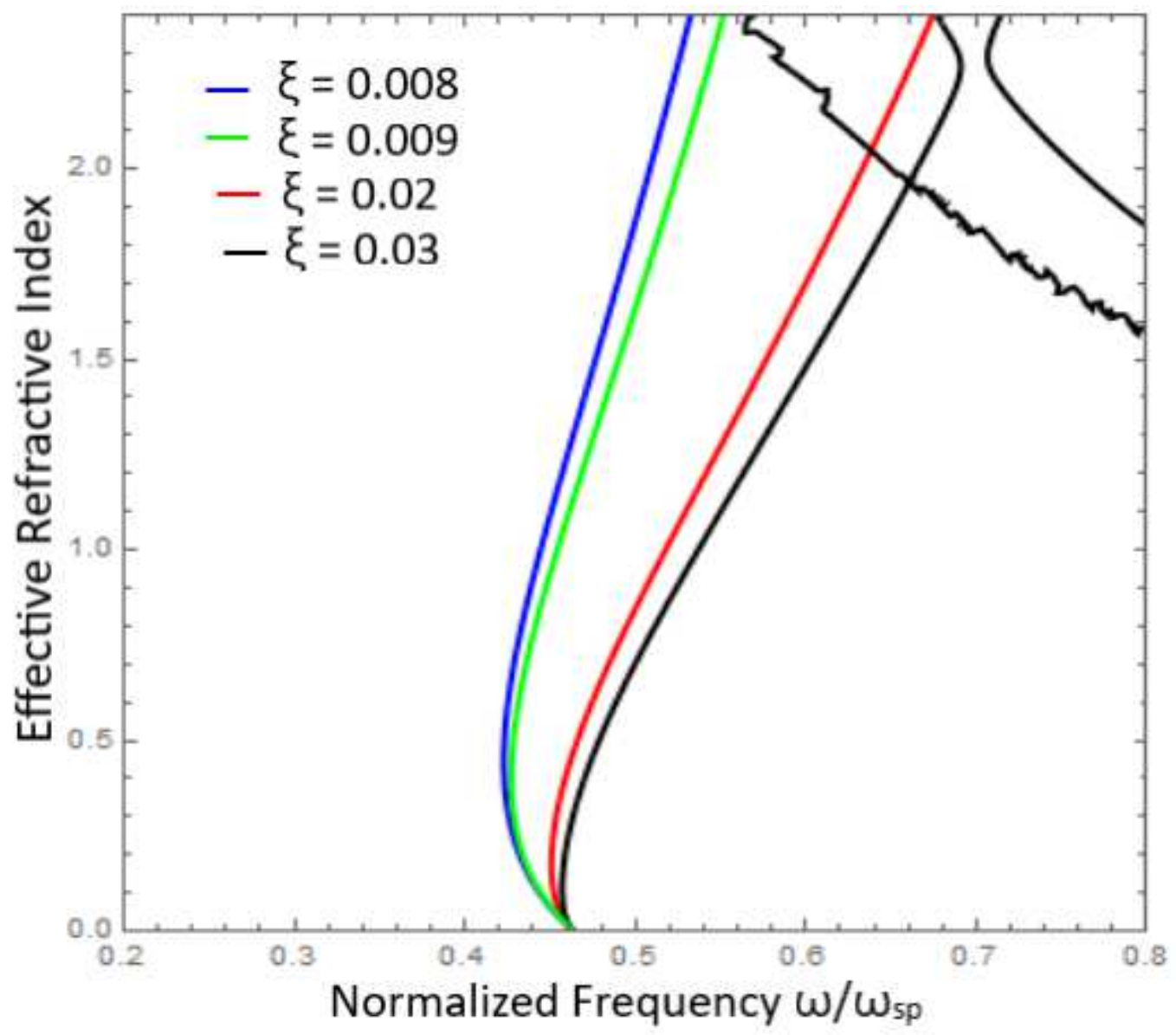

Figure 9

Effect of chiral strength on effective refractive index as function of normalized frequency with $T=300 \mathrm{~K}, \tau$ $=1.6 \mathrm{ps}$, and $\mu \mathrm{c}=0.6 \mathrm{eV}$. 


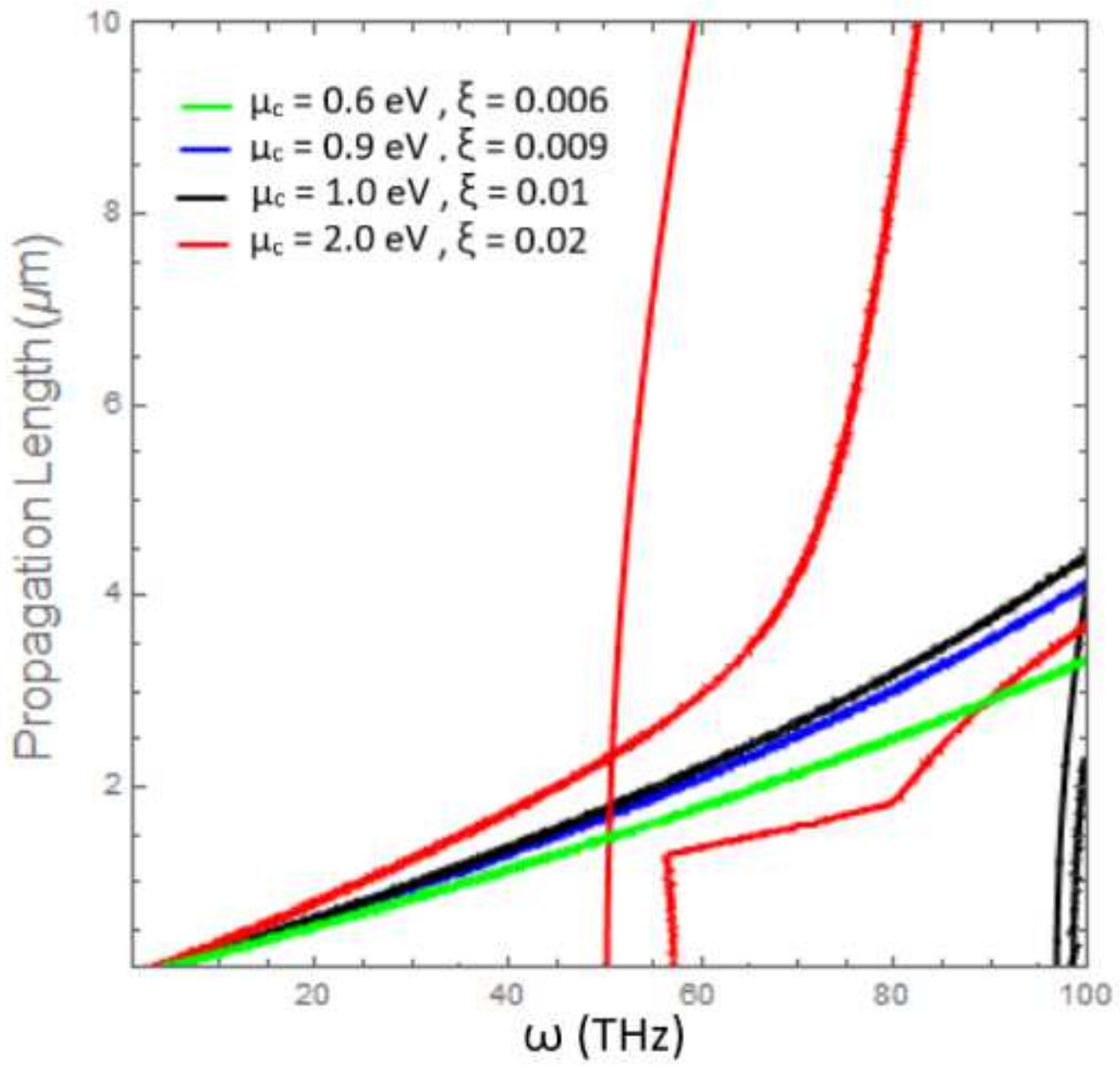

Figure 10

Effect of chiral strength and chemical potential on propagation length of hybrid plasmon modes at CGM interface. 


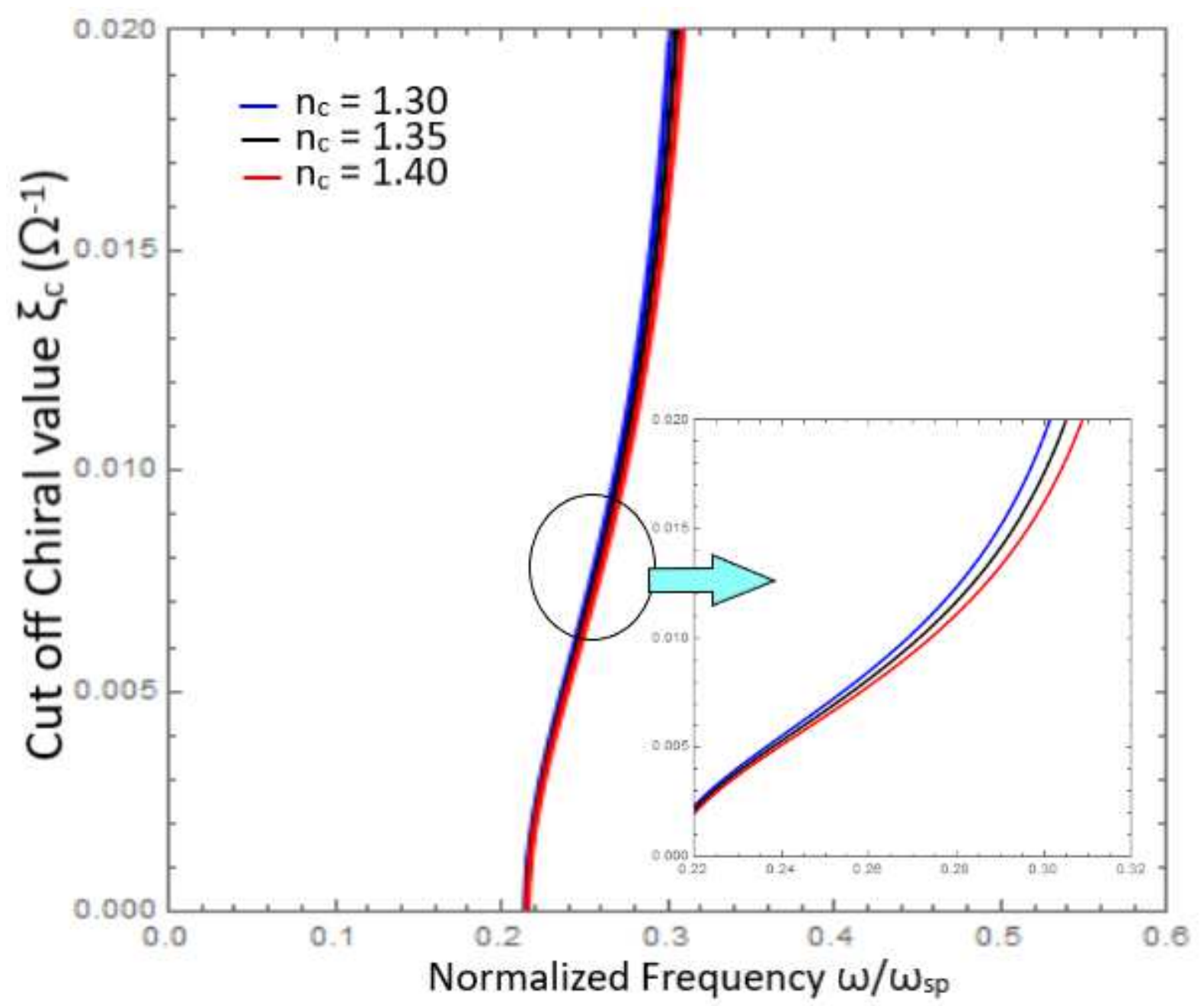

Figure 11

Effect of refractive indices on cutoff chiral strength as function of normalized frequency with $T=300 \mathrm{~K}, \tau$ $=1.6 \mathrm{ps}$, and $\mu \mathrm{c}=0.2 \mathrm{eV}$. 